\title{
Greybody factors for a minimally coupled massless scalar field in Einstein-Born-Infeld dilaton spacetime
}

\author{
Grigoris Panotopoulos \\ CENTRA, Instituto Superior Técnico, Universidade de Lisboa, Av. Rovisco País 1, Lisboa, Portuga* \\ Ángel Rincón \\ Instituto de Física, Pontificia Universidad Católica de Chile, \\ Avenida Vicuña Mackenna 4860, Santiago, Chile $\left.\right|^{\dagger}$
}

(Dated: October 3, 2018)

\begin{abstract}
We have analyzed in detail the propagation of a minimally coupled massless scalar field in the gravitational background of a four-dimensional Einstein-Born-Infeld dilaton charged black hole. We have obtained analytical expressions for the absorption cross section as well as for the decay rate for the scalar field in the aforementioned spacetime, and we have shown graphically its behavior for different values of the free parameters of the theory.
\end{abstract}

PACS numbers: 04.70.Bw, 04.70.Dy, 11.80.-m

\section{INTRODUCTION}

Black holes are objects of paramount importance in gravitational theories. Of particular interest is Hawking's radiation 11. Since it is a manifestation of a quantum effect in curved spacetime, Hawking's radiation has always attracted a lot of attention in the community, despite the fact that up to now it has never been detected in the universe. The greybody factor, or else the absorption cross section, is a frequency dependent factor that measures the modification of the original black body radiation, and thus gives us valuable information about the near horizon structure of black holes [2. Consequently, in the literature exist many works in which the authors have studied the propagation and the relativistic scattering of different kinds of fields, and have analyzed the corresponding greybody factors.

Relativistic scattering of waves has been traditionally studied in asymptotically flat spacetimes without a cosmological constant 3]. However, due to inflation [4, the current cosmic acceleration 5 and the AdS/CFT correspondence 6], asymptotically non-flat spacetimes with a positive or negative cosmological constant have also been studied over the years $7+12$. In 13, however, the authors have found black hole solutions in three and four dimensions that are neither asymptotically flat nor asymptotically (anti) de Sitter. In those works the model is described by the Einstein-Born-Infeld dilaton action. Originally the Born-Infeld non-linear electrodynamics was introduced in the 30's in order to obtain a finite self-energy of point-like charges 14. During the last decades this type of action reappears in the open sector of superstring theories 15] as it describes the dynamics of D-branes 16]. Furthermore, in the closed sector of all superstring theories at the massless level the graviton is accompanied by

*Electronic address: grigorios.panotopoulos@tecnico.ulisboa.pt

${ }^{\dagger}$ Electronic address: arrincon@uc.cl the dilaton that determines the string coupling constant. Since superstring theory is so far the only consistent theory of quantum gravity, it is more than natural to study the greybody factors of black hole solutions obtained in the framework of Einstein-Born-Infeld dilaton models.

In this work we wish to find analytical expressions for the reflection coefficient, the absorption cross-section and the decay rate for a minimally coupled massless scalar field in a four-dimensional Einstein-Born-Infeld dilaton spacetime. Our work is organized as follows: After this introduction, we present the model and the wave equation in the next section. In section 3 we obtain exact solution of the radial equation in terms of hypergeometric functions, and we compute the reflection coefficient as well as the absorption cross-section and the decay rate in section 4. Finally, we conclude our work in the last section.

\section{WAVE EQUATION OF A MASSLESS SCALAR FIELD IN THE EISTEIN-BORN-INFELD DILATON SPACETIME}

Our starting point is the model considered in the second paper of [13] described by the action

$$
\begin{array}{r}
S=\int d^{4} x \sqrt{-g}\left[R-2(\nabla \phi)^{2}-V(\phi)+\right. \\
\left.4 \gamma e^{-2 \kappa \phi}(1-\sqrt{1+Y})\right]
\end{array}
$$

where

$$
Y=\frac{F_{\mu \nu} F^{\mu \nu}}{2 \gamma}
$$

with the scalar field $\phi$ being the dilaton, $V(\phi)$ its potential, $\gamma$ the Born-Infeld parameter, $\kappa$ the dilaton coupling constant, and $F_{\mu \nu}$ the electromagnetic field strength. The solution for the dilaton is given by [13]

$$
\phi(r)=\frac{\kappa}{1+\kappa^{2}} \ln (b r-c)
$$


where $b, c$ are constants of integration. In the following we set for convenience and without loss of generality $b=$ 1 and $c=0$. On the other hand the line element for the metric is given by 13 .

$$
d s^{2}=-h(r) d t^{2}+h(r)^{-1} d r^{2}+e^{2 \kappa \phi} d \Omega^{2}
$$

The dilaton potential is taken to be either $V(\phi)=0$ or a Liouville type potential $V(\phi)=2 \Lambda e^{-2 \kappa \phi}$. Since the model is string inspired, in the following we shall consider the string coupling case $\kappa=1$, in which $h(r)$ is given either by 13

$$
h(r)=2 r\left(1-2 H-\frac{r_{0}}{2 r}\right)
$$

if $V(\phi)=0$, or by 13

$$
h(r)=2 r\left(1-2 H-\Lambda-\frac{r_{0}}{2 r}\right)
$$

if $V(\phi)=2 \Lambda e^{-2 \phi}$. Therefore in both cases the function $h(r)$ turns out to be linear in $r$ irrespectively of the dilaton potential, namely $h(r)=r / L-r_{0}$ where $r_{0}, L$ are constants. The constant $r_{0}$ is related to the mass of the black hole [13], $r_{0}=4 M$, while the length scale $L$ is given by

$$
L^{-1}=2(1-\Lambda-2 H)
$$

where the constant $H$ is given by 13

$$
H=-\gamma+\sqrt{\gamma\left(Q^{2}+\gamma\right)}
$$

and the charge $Q$ of the black hole is given by 13

$$
Q^{2}=\frac{1+\sqrt{1+16 \gamma^{2}}}{8 \gamma}
$$

There is a single event horizon $r_{H}=L r_{0}$, and therefore the line element takes the form

$$
d s^{2}=-h(r) d t^{2}+h(r)^{-1} d r^{2}+r d \Omega^{2}
$$

where $h(r)=\left(r-r_{H}\right) / L$. Therefore the metric is characterized by two length scales $L, r_{H}$ which are given functions of the three free parameters of the model, namely the black hole mass $M$, the Born-Infeld parameter $\gamma$, and the mass scale $\Lambda$ in dilaton's potential. Note that $r_{H}$ depends on all three free parameters, while $L$ does not depend on the mass of the black hole.

Now we consider a minimally coupled massless scalar field $\Psi$ in the above background. The equation of motion is the standard Klein-Gordon equation

$$
\frac{1}{\sqrt{-g}} \partial_{\mu}\left(\sqrt{-g} g^{\mu \nu} \partial_{\nu} \Psi\right)=0
$$

and using the ansatz $\Psi(t, r, \theta, \phi)=e^{-i \omega t} R(r) Y_{l}^{m}(\theta, \phi)$, where $Y_{l}^{m}$ are the usual spherical harmonics, we obtain the radial equation

$$
R^{\prime \prime}+\left(\frac{h^{\prime}}{h}+\frac{1}{r}\right) R^{\prime}+\left(\frac{\omega^{2}}{h^{2}}-\frac{l(l+1)}{r h}\right) R=0
$$

To see the potential that the scalar field feels we define new variables as follows

$$
\begin{aligned}
R & =\frac{\psi}{\sqrt{r}} \\
x & =\int \frac{d r}{h(r)}=L \ln \left(\frac{r-r_{H}}{d}\right)
\end{aligned}
$$

where $x$ is the so called tortoise coordinate and $d$ is a constant of integration which will be taken as unity. We recast the equation for the radial part into a Schrödingerlike equation of the form

$$
\frac{d^{2} \psi}{d x^{2}}+\left(\omega^{2}-V(x)\right) \psi=0
$$

Therefore we obtain for the effective potential barrier the expression

$$
V(r)=h(r)\left(\frac{l(l+1)}{r}+\frac{h^{\prime}(r)}{2 r}-\frac{h(r)}{4 r^{2}}\right)
$$

which can be simplified to be

$$
V(r)=V_{0}-\frac{r_{H} l(l+1)}{L r}-\frac{r_{H}^{2}}{4 L^{2} r^{2}}
$$

where the constant term is given by $V_{0}=(\operatorname{Ll}(l+1)+$ $1 / 4) / L^{2}$. The effective potential barrier as a function of the radial distance can be seen in Fig. 1 and 2 below for $l=1$ and $l=0$ respectively, and three different cases, namely $\gamma=1, \Lambda=0$, and $M=1.1,1.75,2.25$.

Since at the horizon the effective potential vanishes, the general solution for the function $\psi$ close to the horizon (where $\omega^{2} \gg V(x)$ ) is given by

$$
\psi(x)=C_{+} e^{i \omega x}+C_{-} e^{-i \omega x}
$$

while requiring purely ingoing solution we set $C_{-}=0$, and thus the solution becomes

$$
\psi(x)=C_{+} e^{i \omega x}
$$

On the other hand, it is easy to check that at large $r$ (or at large $x$, since when $r \gg r_{H}, r \simeq e^{x / L}$ ) the potential tends to the constant $V_{0}$, and therefore defining $\Omega \equiv \sqrt{\omega^{2}-V_{0}}$ the solution for $\psi$ is given by

$$
\psi(x)=A_{+} e^{i \Omega x}+A_{-} e^{-i \Omega x} .
$$

Therefore the far-field solution expressed in the tortoise coordinate $x$ takes the form of ingoing and outgoing plane waves provided that $\omega^{2}>V_{0}$.

\section{SOLUTION OF THE RADIAL DIFFERENTIAL EQUATION}

\section{A. Solution in the far-field region}

In the far-field region $r \gg r_{H} h(r) \sim r / L$ and the radial equation becomes

$$
R^{\prime \prime}(r)+\frac{2}{r} R^{\prime}(r)+\frac{q}{r^{2}} R(r)=0
$$




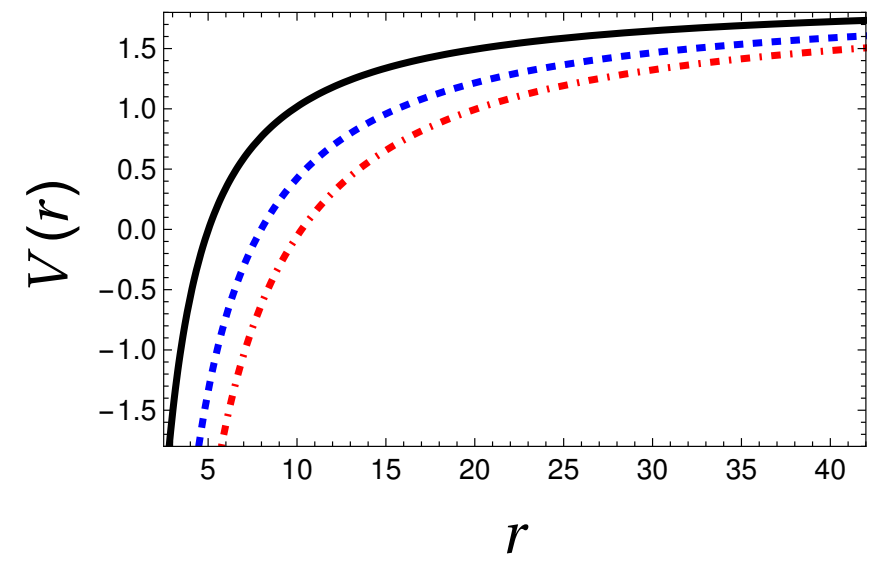

FIG. 1: Effective potential versus $r$ for $l=1, \gamma=1, \Lambda=0$ and for $M=1.1$ (solid black line), $M=1.75$ (dashed blue line) and $M=2.25$ (dot-dashed red line).

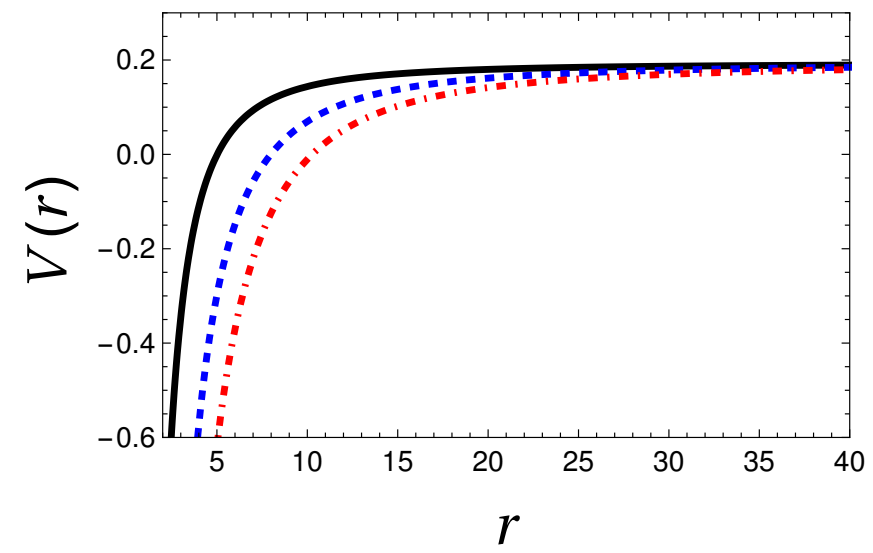

FIG. 2: Same as in Fig. 1, but for $l=0$.

where $q=(\omega L)^{2}-L l(l+1)$ or

$$
r^{2} R^{\prime \prime}(r)+2 r R^{\prime}(r)+q R(r)=0
$$

which is Euler's equation. We seek solutions of the form $R(r) \sim r^{\rho}$ and the power $\rho$ satisfies the algebraic equation

$$
\rho^{2}+\rho+q=0
$$

The determinant is found to be $\Delta=-4 L^{2}\left(\omega^{2}-V_{0}\right)$, and the algebraic equation above admits two roots given by

$$
\rho_{ \pm}=-\frac{1}{2} \pm \sqrt{\frac{1}{4}-q}
$$

and they are real when $1 / 4 \geq q$, while when $1 / 4<q$ the roots are complex. Therefore the solution in the far-field region reads

$$
R_{F F}=D_{1}\left(\frac{r}{r_{H}}\right)^{\rho_{-}}+D_{2}\left(\frac{r}{r_{H}}\right)^{\rho_{+}}
$$

where $D_{1}, D_{2}$ are two arbitrary coefficients. In terms of the tortoise coordinate $x$, the radial part takes the form of ingoing and outgoing plane waves only when the two roots above are complex as follows

$$
R_{F F}=\frac{1}{\sqrt{r}}\left(\frac{D_{1}}{r_{H}^{\rho_{-}}} e^{-i \Im\left(\rho_{+}\right) \frac{x}{L}}+\frac{D_{2}}{r_{H}^{\rho_{+}}} e^{i \Im\left(\rho_{+}\right) \frac{x}{L}}\right)
$$

with $\Im\left(\rho_{+}\right)$being the imaginary part of $\rho_{+}$defined in Eq. (24). It can also be seen from the solution of the Schrödinger-like equation at large $x$, as we already mentioned in the end of Section II. From the above it is clear that $D_{1}, D_{2}$ represent the ingoing and outgoing waves respectively. Therefore the reflection coefficient is defined to be $\mathcal{R}=\left|D_{1} / D_{2}\right|^{2}$, and in the following we shall consider the case where $q>1 / 4$ or $\omega^{2}>V_{0}$. In this case the roots are given by

$$
\rho_{ \pm}=-\frac{1}{2} \pm i \sqrt{-\frac{1}{4}+q}
$$

Note that in the far-field solution for the radial part there is also a decaying amplitude $1 / \sqrt{r}$, but since it is present in both terms of the solution, it drops from the final expression upon taking the ratio to compute the reflection coefficient.

\section{B. Exact solution in terms of hypergeometric functions}

Next we find an exact solution of the radial equation (12) in terms of hypergeometric functions introducing $z=1-r_{H} / r$. The new equation for $z$ reads

$$
z(1-z) R_{z z}+(1-z) R_{z}+\left(\frac{A}{z}+\frac{B}{-1+z}\right) R=0
$$

where $A=(\omega L)^{2}, B=-(\omega L)^{2}+L l(l+1)$. To get rid of the poles we set

$$
R=z^{\alpha}(1-z)^{\beta} F
$$

where now $F$ satisfies the following differential equation

$$
\begin{aligned}
& z(1-z) F_{z z}+[1+2 \alpha-(1+2 \alpha+2 \beta) z] F_{z} \\
&+\left(\frac{\bar{A}}{z}+\frac{\bar{B}}{-1+z}-C\right) F=0
\end{aligned}
$$

and the new constants are given by

$$
\begin{aligned}
& \bar{A}=A+\alpha^{2} \\
& \bar{B}=B+\beta-\beta^{2} \\
& C=(\alpha+\beta)^{2}
\end{aligned}
$$

Demanding that $\bar{A}=0=\bar{B}$ we obtain the Gauss' hypergeometric equation

$$
z(1-z) F_{z z}+[c-(1+a+b) z] F_{z}-a b F=0
$$


and we determine the parameters $\alpha, \beta$ as follows

$$
\begin{aligned}
& \alpha=i \omega L \\
& \beta=\frac{1}{2}+i \sqrt{(\omega L)^{2}-L l(l+1)-\frac{1}{4}}
\end{aligned}
$$

Finally the three parameters of Gauss' equation are given by

$$
\begin{aligned}
& c=1+2 \alpha \\
& a=\alpha+\beta \\
& b=\alpha+\beta
\end{aligned}
$$

Note that the parameters $a, b, c$ satisfy the condition $c-$ $a-b=1-2 \beta$. Therefore the solution for the radial part is given by

$$
R(z)=D z^{\alpha}(1-z)^{\beta} F(a, b ; c ; z)
$$

where $D$ is an arbitrary coefficient, and the hypergeometric function can be expanded in a Taylor series as follows

$$
F(a, b ; c ; z)=1+\frac{a b}{c} z+\ldots
$$

Note that the above solution for the choice of $\alpha=i \omega L$ reproduces the purely ingoing solution at the horizon (19), as it can be seen from the fact that close to the horizon $(z \rightarrow 0)$, the radial part becomes $R(z) \simeq D z^{\alpha}$, and the parameter $\mathrm{z}$ can be written approximately $z \simeq$ $\left(r-r_{H}\right) / r_{H}=e^{x / L} / r_{H}$.

\section{Matching of the solutions}

In order to match with the far field solution obtained earlier (where now $z \rightarrow 1$ ) we use the transformation [17]

$$
\begin{aligned}
F(a, b ; c ; z)= & \frac{\Gamma(c) \Gamma(c-a-b)}{\Gamma(c-a) \Gamma(c-b)} \times \\
& F(a, b ; a+b-c+1 ; 1-z)+ \\
(1-z)^{c-a-b} & \frac{\Gamma(c) \Gamma(a+b-c)}{\Gamma(a) \Gamma(b)} \times \\
& F(c-a, c-b ; c-a-b+1 ; 1-z)
\end{aligned}
$$

and therefore the radial part as $z \rightarrow 1$ reads

$$
\begin{aligned}
R(z \rightarrow 1)= & D(1-z)^{\beta} \frac{\Gamma(1+2 \alpha) \Gamma(1-2 \beta)}{\Gamma(1+\alpha-\beta) \Gamma(1+\alpha-\beta)} \\
& +D(1-z)^{1-\beta} \frac{\Gamma(1+2 \alpha) \Gamma(-1+2 \beta)}{\Gamma(\alpha+\beta) \Gamma(\alpha+\beta)}
\end{aligned}
$$

Note that $-\beta=\rho_{-}$and $\beta-1=\rho_{+}$, and since $z=$ $1-\left(r_{H} / r\right)$ the radial part $R(r)$ for $r \gg r_{H}$ can be written down as follows

$$
\begin{aligned}
R(r)=D & \frac{\Gamma(1+2 \alpha) \Gamma(1-2 \beta)}{\Gamma(1+\alpha-\beta) \Gamma(1+\alpha-\beta)}\left(\frac{r}{r_{H}}\right)^{\rho_{-}} \\
& +D \frac{\Gamma(1+2 \alpha) \Gamma(-1+2 \beta)}{\Gamma(\alpha+\beta) \Gamma(\alpha+\beta)}\left(\frac{r}{r_{H}}\right)^{\rho_{+}}
\end{aligned}
$$

Finally upon comparison we express $D_{1}, D_{2}$ in terms of $D$ as follows

$$
\begin{aligned}
& D_{1}=D \frac{\Gamma(1+2 \alpha) \Gamma(1-2 \beta)}{\Gamma(1+\alpha-\beta) \Gamma(1+\alpha-\beta)} \\
& D_{2}=D \frac{\Gamma(1+2 \alpha) \Gamma(-1+2 \beta)}{\Gamma(\alpha+\beta) \Gamma(\alpha+\beta)}
\end{aligned}
$$

\section{THE ABSORPTION CROSS-SECTION AND DISCUSSION OF THE RESULTS}

The reflection coefficient is given by $\mathcal{R}=\left|D_{1} / D_{2}\right|^{2}$, and according to the previous results is computed to be

$$
\mathcal{R}=\left|\frac{\Gamma(\alpha+\beta)^{2} \Gamma(1-2 \beta)}{\Gamma(1+\alpha-\beta)^{2} \Gamma(-1+2 \beta)}\right|^{2}
$$

and using the following identities for the $\Gamma$ function [8]

$$
\begin{aligned}
|\Gamma(i y)|^{2} & =\frac{\pi}{y \sinh (\pi y)} \\
\left|\Gamma\left(\frac{1}{2}+i y\right)\right|^{2} & =\frac{\pi}{\cosh (\pi y)}
\end{aligned}
$$

we obtain the final expression

$$
\mathcal{R}=\frac{\cosh ^{2}\left[\pi\left[L \omega-\sqrt{-l(l+1) L+L^{2} \omega^{2}-\frac{1}{4}}\right]\right]}{\cosh ^{2}\left[\pi\left[L \omega+\sqrt{-l(l+1) L+L^{2} \omega^{2}-\frac{1}{4}}\right]\right]}
$$

The reflection coefficient depends on $L$ only, and not on $r_{H}$. Therefore it does not depend on the mass of the black hole. As a function of the frequency it can be seen in Fig. 3 and 4 below for $l=0$. It always starts at 1 and monotonically decreases to zero very fast. In Fig. 3 we have set $\Lambda=0$ and we have considered three different values for $\gamma=0.5,1,2$, while in Fig. 4 we have set $\gamma=1$ and we have considered three different values for $\Lambda=0.001,0.01,0.1$. The curves move to the right as we decrease $L$ (decrease $\Lambda$ or increase $\gamma$ ) due to the inequality found before $\omega>\sqrt{4 l^{2} L+4 l L+1} / 2 L$.

The absorption cross section is given by the optical theorem [2, 18]

$$
\sigma_{a b s}=\Lambda_{l} \frac{(1-\mathcal{R})}{\omega^{2}}
$$

where $\Lambda_{l}=(2 l+1) \pi$ and therefore the full expression is given by

$$
\sigma_{a b s}=\frac{\Lambda_{l}}{\omega^{2}}\left[1-\frac{\cosh ^{2}[\pi[L \omega-\Im(\beta)]]}{\cosh ^{2}[\pi[L \omega+\Im(\beta)]]}\right]
$$

where $\Im(\beta)=\Im\left(\rho_{+}\right)$denotes the imaginary part of $\beta$ defined at Eq. (36). The greybody factor as a function of $\omega$ is shown in Fig. 5 and 6 below for $l=0$. We have considered the same values for $\gamma$ and $\Lambda$ as in Fig. 3 and 4 


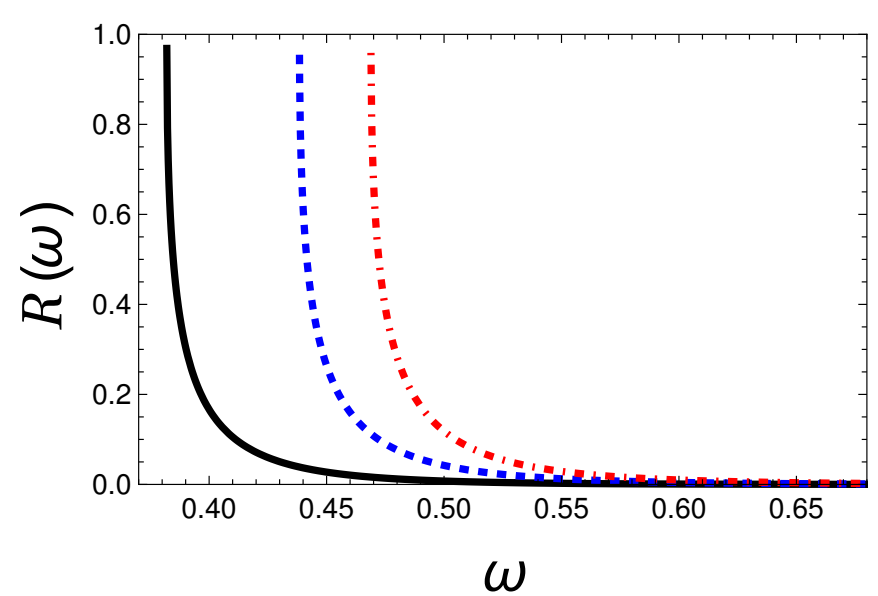

FIG. 3: Reflection coefficient versus $\omega$ for $l=0, \Lambda=0$ and from left to right $\gamma=0.5, \gamma=1$ and $\gamma=2$.

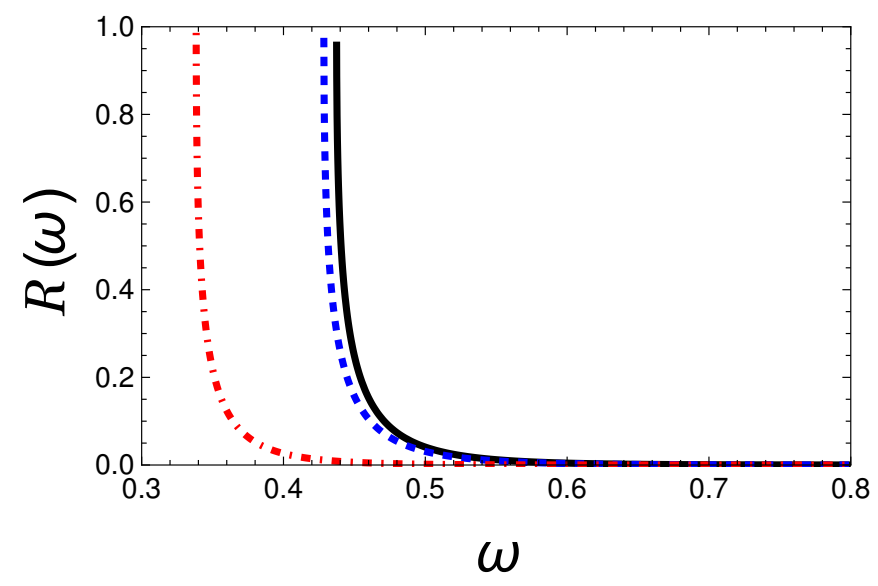

FIG. 4: Reflection coefficient versus $\omega$ for $l=0, \gamma=1$, and from left to right $\Lambda=0.1, \Lambda=0.01$ and $\Lambda=0.001$.

respectively. First the absorption cross section increases with $\omega$ until it reaches a maximum, and then tends to zero monotonically but not as fast as the reflection coefficient. Due to the same inequality, as we decrease $L$ the curves move to the right, while at the same time the maximum value gets lower.

Since the flux spectrum emitted by the black hole is given by 9

$$
\frac{d N(\omega)}{d t}=\sum_{l} \frac{\sigma_{l}(\omega)}{e^{\omega / T_{H}}-1} \frac{d^{3} k}{(2 \pi)^{3}}
$$

the decay rate is defined to be [8]

$$
\Gamma_{\text {decay }}=\frac{\sigma_{a b s}}{e^{\omega / T_{H}}-1}
$$

where the Hawking temperature is computed to be $T_{H}=$
$1 /(4 \pi L)$ [13. Thus, the full expression is given by

$$
\Gamma_{\text {decay }}=\frac{\Lambda_{l}}{\omega^{2}}\left[\frac{e^{2 \pi \sqrt{-4 L\left(l^{2}+l-L \omega^{2}\right)-1}}-1}{\left[e^{\pi \sqrt{-4 L\left(l^{2}+l-L \omega^{2}\right)-1}+2 \pi L \omega}+1\right]^{2}}\right]
$$

and as a function of the frequency it can be seen in Fig. 7 and 8 below for $l=0$. We have considered the same values for $\gamma$ and $\Lambda$ as in Fig. 3 and 4 respectively. The decay rate reaches a maximum and then quickly decays to zero. As we decrease $\Lambda$ or increase $\gamma$ the curves move to the right and at the same time the maximum value decreases too, precisely as in the greybody factor case.

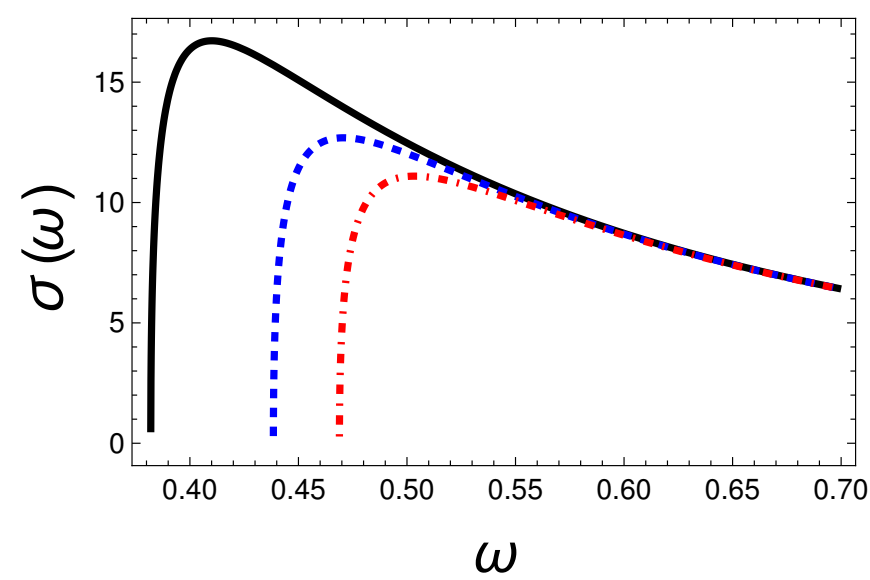

FIG. 5: Absorption cross section as a function of $\omega$ for $l=0$ and values for $\gamma, \Lambda$ as in Fig. 3 .

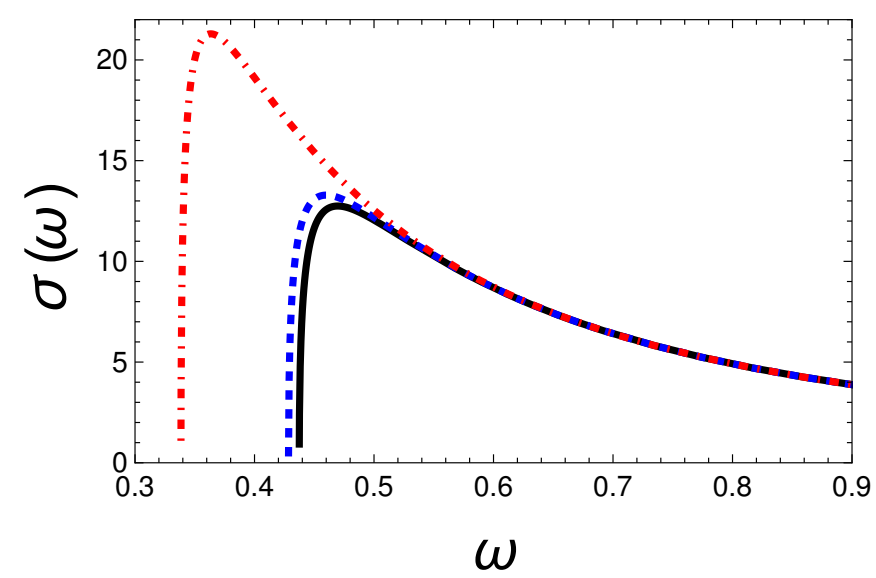

FIG. 6: Absorption cross section as a function of $\omega$ for $l=0$ and values for $\gamma, \Lambda$ as in Fig. 4 . 


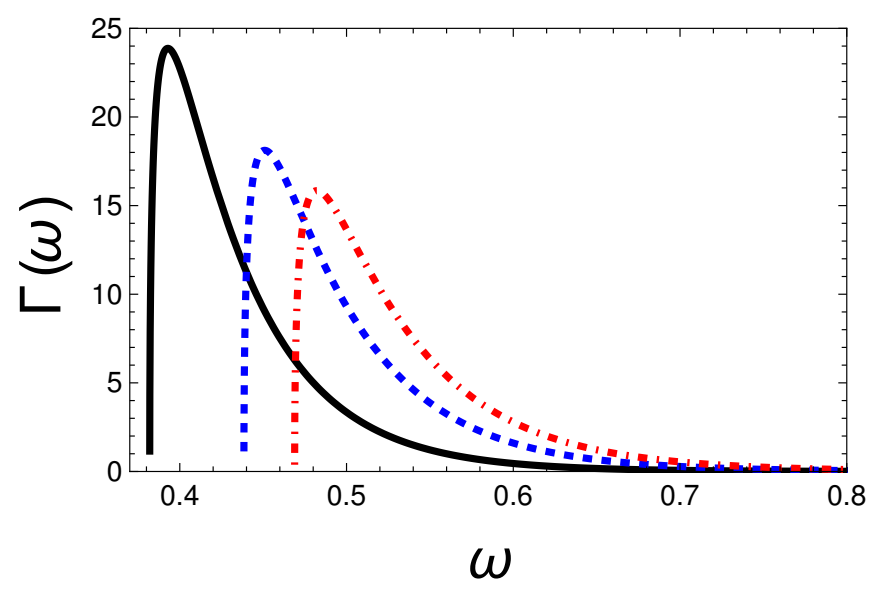

FIG. 7: Decay rate as a function of $\omega$ for $l=0$ and values for $\gamma, \Lambda$ as in Fig. 3. Note that the vertical axis is scaled $1: 10^{-3}$.

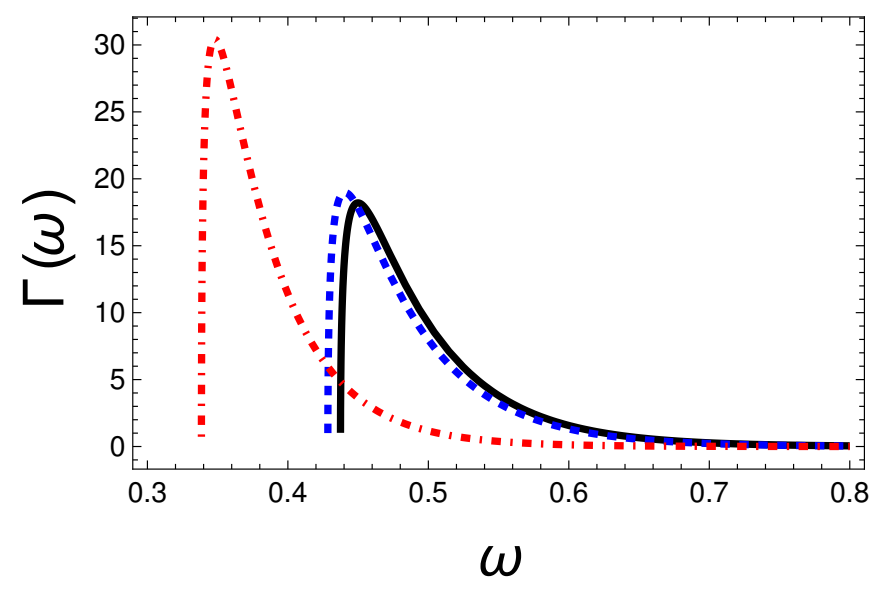

FIG. 8: Decay rate as a function of $\omega$ for $l=0$ and values for $\gamma, \Lambda$ as in Fig. 4. Note that the vertical axis is scaled $1: 10^{-3}$.

\section{CONCLUSIONS}

To summarize, in this article we have analyzed the greybody factors for a minimally coupled massless scalar scalar field in a four-dimensional Einstein-Born-Infeld dilaton charged black hole background. Since the model is string inspired we have considered the string coupling case $\kappa=1$. We have found exact solution of the radial equation in terms of the hypergeometric functions, and we have obtained analytical expressions for the effective barrier potential, the reflection coefficient, the absorption cross section as well as the decay rate. We have shown in figures how the above quantities behave for different values of the free parameters of the theory. Our results are qualitatively similar to those for a three-dimensional Einstein-Maxwell dilaton black hole.

\section{Acknowledgments}

We wish to thank the anonymous reviewers for valuable comments and suggestions. The author G.P. acknowledges the support from "Fundação para a Ciência e Tecnologia". The work of A.R. was supported by the CONICYT-PCHA/Doctorado Nacional/2015-21151658.
[1] S. W. Hawking, Nature 248 (1974) 30;

S. W. Hawking, Commun. Math. Phys. 43 (1975) 199 Erratum: [Commun. Math. Phys. 46 (1976) 206].

[2] P. Kanti and J. March-Russell, Phys. Rev. D 66 (2002) 024023 hep-ph/0203223.

[3] C. Doran, A. Lasenby, S. Dolan, and I. Hinder, Phys. Rev. D 71, 124020 (2005);

S. Dolan, C. Doran, and A. Lasenby, Phys. Rev. D 74, 064005 (2006);

L. C. B. Crispino, E. S. Oliveira, A. Higuchi, and G. E.

A. Matsas, Phys. Rev. D 75, 104012 (2007);

S. R. Dolan, Classical Quantum Gravity 25, 235002 (2008);

L. C. B. Crispino, S. R. Dolan, and E. S. Oliveira, Phys. Rev. Lett. 102, 231103 (2009);

L. C. B. Crispino, S. R. Dolan, and E. S. Oliveira, Phys. Rev. D 79, 064022 (2009);

L. B. Crispino, A. Higuchi, and E. S. Oliveira, Phys. Rev. D 80, 104026 (2009).

[4] A. H. Guth, Phys. Rev. D 23, 347 (1981).

[5] A. G. Riess et al. Astron. J. 116, 1009 (1998);
S. Perlmutter et al., Astrophys. J. 517, 565 (1999).

[6] J. M. Maldacena, Int. J. Theor. Phys. 38 (1999) 1113 [Adv. Theor. Math. Phys.A. Sheykhi, N. Riazi and M. H. Mahzoon, Phys. Rev. D 74 (2006) 044025 hepth/0605043. 2 (1998) 231] hep-th/9711200;

I. R. Klebanov, hep-th/0009139

[7] D. Birmingham, I. Sachs and S. Sen, Phys. Lett. B 413 (1997) 281 hep-th/9707188;

Y. S. Myung, Mod. Phys. Lett. A 18 (2003) 617 hepth/0201176.

[8] S. Fernando, Gen. Rel. Grav. 37 (2005) 461, hepth/0407163.

[9] L. C. B. Crispino, A. Higuchi, E. S. Oliveira and J. V. Rocha, Phys. Rev. D 87 (2013) 104034 arXiv:1304.0467 [gr-qc]].

[10] P. Kanti, T. Pappas and N. Pappas, Phys. Rev. D 90 (2014) no.12, 124077 arXiv:1409.8664 [hep-th]]; T. Pappas, P. Kanti and N. Pappas, Phys. Rev. D 94 (2016) no.2, 024035 arXiv:1604.08617 [hep-th].

[11] G. Panotopoulos and Á. Rincón, arXiv:1611.06233 [hepth]. 
[12] J. Ahmed and K. Saifullah, arXiv:1610.06104 [gr-qc].

[13] R. Yamazaki and D. Ida, Phys. Rev. D 64 (2001) 024009 doi:10.1103/PhysRevD.64.024009 gr-qc/0105092; A. Sheykhi, N. Riazi and M. H. Mahzoon, Phys. Rev. D 74 (2006) 044025 hep-th/0605043.

[14] M. Born and L. Infeld, Proc. Roy. Soc. Lond. A 144 (1934) 425.

[15] M. B. Green, J. H. Schwarz and E. Witten, Superstring Theory, Vol. 1 \& 2, Cambridge Monographs on Mathematical Physics;

J. Polchinski, String Theory, Vol. 1 \& 2, Cambridge
Monographs on Mathematical Physics.

[16] C. V. Johnson, D-Branes, Cambridge Monographs on Mathematical Physics;

B. Zwiebach, A First Course in String Theory, Cambridge University Press.

[17] M. Abramowitz and I. Stegun, Handbook of Mathematical Functions (Academic, New York, 1966).

[18] S. S. Gubser, I. R. Klebanov and A. A. Tseytlin, Nucl. Phys. B 499 (1997) 217 hep-th/9703040. 